\title{
Use of the Prevena Incision Management System as a potential solution for high-risk, complicated perineal wounds
}

\author{
J. $\operatorname{Mino}^{1} \cdot$ F. H. Remzi ${ }^{1}$
}

Received: 4 March 2016/Accepted: 28 April 2016/Published online: 11 May 2016

(C) Springer-Verlag Italia Srl 2016

Perineal wound complications are common following abdominoperineal resection. Risk factors include pre-operative radiation, indication for surgery, diabetes, obesity, and smoking. Wound management has evolved from open wound packing to primary closure with closed-suction drains. Nonetheless, complications remain high with breakdown and/or infection rates as high as $30 \%$ or more. This can result in prolonged hospital stay, readmission, wound care needs, and significant medical costs. Adjuvant therapies have evolved, including the use of vacuum-assisted wound devices, although these result in increased healing times compared to primary closure due to the open nature of the wound with these devices. The use of closedsuction systems over a primarily closed incision has recently been developed. However, large series are lacking, in part likely due to the difficulty of application in this region. We present here the application of the Prevena Incision Management System for use in the perineal incisions due to its ease of application, low cost, and patient tolerance. This is our first abdominoperineal resection case on which we used the Prevena system, with excellent results. Ease of application was enhanced due to the premade nature of the system. Currently, we are further evaluating the efficacy of this system in decreasing wound complications, in hopes of improved outcomes in these high-risk situations, where treatment otherwise involves major reconstruction, tissue flaps, or other costly and involved procedures.

\section{Compliance with ethical standards}

Conflict of interest The authors declare that they have no conflict of interest.

Ethical approval This study complied with all ethical standards.

Informed consent Informed consent was obtained from the patient.
Electronic supplementary material The online version of this article (doi:10.1007/s10151-016-1490-y) contains supplementary material, which is available to authorized users.

\author{
J. Mino \\ minoj@ccf.org \\ 1 Cleveland Clinic, Cleveland, OH, USA
}

